
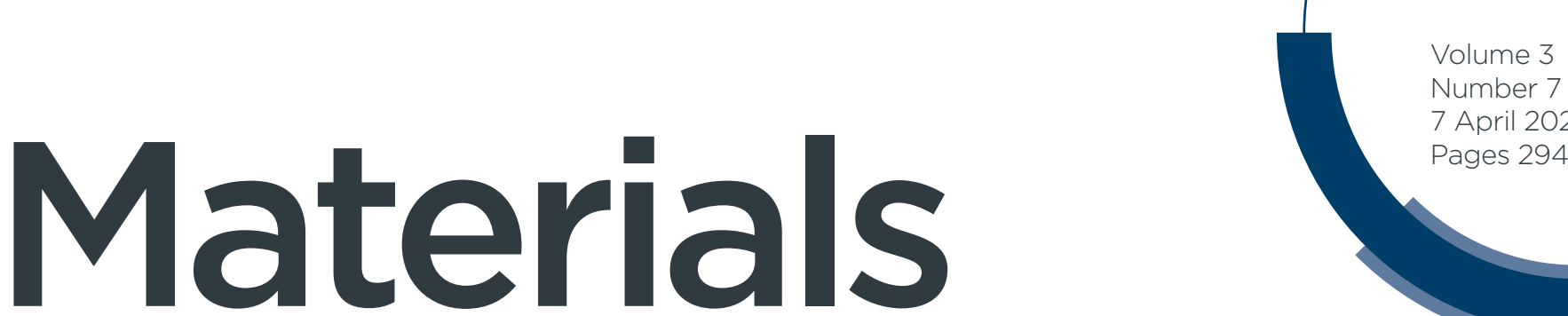

Number 7

7 April 2022

Pages 2949-3334

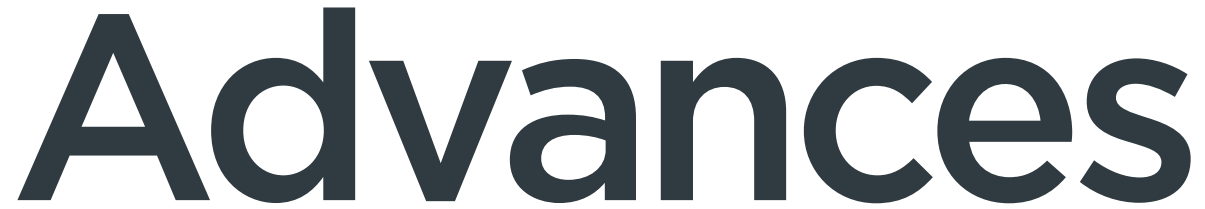

rsc.li/materials-advances

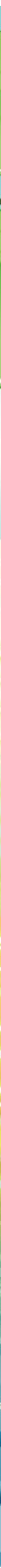

ISSN 2633-5409

ROYAL SOCIETY OF CHEMISTRY

\section{PAPER}

Junko Aimi et al.

Fabrication of solution-processable OFET memory using

a nano-floating gate based on a phthalocyanine-cored star-shaped polymer 
Check for updates

Cite this: Mater. Adv., 2022, 3,3128

Received 16th November 2021,

Accepted 2nd February 2022

DOI: $10.1039 / \mathrm{d} 1 \mathrm{ma01081f}$

rsc.li/materials-advances

\title{
Fabrication of solution-processable OFET memory using a nano-floating gate based on a phthalocyanine-cored star-shaped polymer $\dagger$
}

\author{
Junko Aimi, (D) *a Takeshi Yasuda, (D) ${ }^{a}$ Chih-Feng Huang, (D) ${ }^{b}$ Masafumi Yoshio (D) ${ }^{a}$ \\ and Wen-Chang Chen (iD ${ }^{c}$
}

\begin{abstract}
Solution-processed organic field-effect transistor (OFET) memory devices are fabricated using a blend film of 6,13-bis(triisopropylsilylethynyl)pentacene and phthalocyanine-cored star-shaped polystyrene. A highly crystalline organic semiconductor thin film was obtained on the star-shaped polymer with charge-trapping sites via a one-pot spin-coating process through vertical phase separation, which is advantageous for OFET memory device applications. The resultant OFET device demonstrated a charge carrier mobility of $0.10 \mathrm{~cm}^{2} \mathrm{~V}^{-1} \mathrm{~s}^{-1}$ and an on/off current ratio of $10^{6}$. Upon application of a gate bias, a substantial reversible threshold shift was observed, along with long charge-retention ability, thereby confirming the memory characteristics of the device.
\end{abstract}

\section{Introduction}

Nonvolatile organic field-effect transistor (OFET) memory devices have attracted much attention because of their easily integrated structure and nondestructive readout. ${ }^{1,2}$ They are regarded as a promising technology to integrate switching capacity and memory into various organic electronic devices such as sensors, displays, and radio-frequency identification tags. Meeting the recent demand for flexible, wearable, and large-area electronic devices via an easy and low-cost production process will necessitate the development of solution-processable OFET memories. ${ }^{3-5}$ However, OFET memory often requires complicated and multistep fabrication processes because it contains multilayers of thin films, including an organic semiconductor for charge transport and a memory layer for charge trapping as well as a tunneling layer for charge insertion/extraction and a blocking layer for charge retention. ${ }^{6-8}$ In addition, the active layer of an organic semiconductor in OFET memory devices is normally prepared by vacuum deposition to achieve high charge carrier mobility. Therefore, the preparation of OFET

\footnotetext{
${ }^{a}$ Research Center for Functional Materials, National Institute for Materials Science (NIMS), 1-2-1 Sengen, Tsukuba, Ibaraki 305-0047, Japan.

E-mail: AIMI.Junko@nims.go.jp

${ }^{b}$ Department of Chemical Engineering, $i$-Center for Advanced Science and Technology (iCAST), National Chung Hsing University, Taichung 40227, Taiwan ${ }^{c}$ Department of Chemical Engineering, National Taiwan University, Taipei 10617, Taiwan

$\dagger$ Electronic supplementary information (ESI) available. See DOI: 10.1039/ d1ma01081f
}

memory devices using simple solution processes is a key challenge. ${ }^{9-14}$

Solution-processable organic semiconductors themselves have been extensively investigated and incorporated into OFET devices. For instance, 6,13-bis(triisopropylsilylethynyl)pentacene (TIPS-pentacene) is a typical small-molecule semiconductor with solubilizing side groups. The charge-carrier mobility in OFET devices is strongly influenced by the crystalline structure and alignment of the organic semiconductors. ${ }^{15}$ To achieve high device performance using a solution process, researchers have studied various methods of preparing thin films of organic semiconductors. Polymer-matrix-assisted phase separation is one of the most promising methods to prepare OFETs with high charge carrier mobility. ${ }^{16-21}$ The mixed polymer increases the wettability of the substrates and decreases the evaporation speed of the solvents to induce crystallization of the organic semiconductor. For instance, an OFET prepared using a spin-coated blend film of TIPS-pentacene and poly $(\alpha$-methylstyrene) exhibited a charge carrier mobility as high as $0.54 \mathrm{~cm}^{2} \mathrm{~V}^{-1} \mathrm{~s}^{-1} .^{17}$ The selection of an appropriate polymer insulator, ${ }^{22-24}$ polymer molecular weight, ${ }^{17,25,26}$ and processing solvent ${ }^{27-30}$ is important for controlling the crystal growth of organic semiconductors in the polymer matrix. Further advances in the coating process, such as the development of the blade coating technique, ${ }^{25,31,32}$ have led to additional enhancements in OFET device performance.

Here we envision that the method of using a polymer matrix to construct high-performance OFET devices via a solution process can also be applied to OFET memory devices when the matrix polymer is capable of charge storage. Recently, we prepared metallophthalocyanine (MPc)-cored star-shaped 
polystyrene (MSP) and used it as a charge-trapping material in a pentacene-based OFET memory device. ${ }^{33}$ The star polymer with an MPc core is readily soluble in organic solvents, enabling the fabrication of polymer films with a smooth surface via simple spin-coating onto a Si substrate. The MSP films exhibited a unique three-dimensional morphology, where the MPc cores were distributed and isolated by the surrounding arm-polymer matrix. A bottom-gate top-contact OFET memory device was fabricated via vacuum deposition of pentacene onto the polymer film, followed by deposition of Au electrodes. When a negative gate voltage was applied to the device, hole charges that accumulated in pentacene migrated to the MPc core, whereas polystyrene arms restrained leakage of the trapped charges to achieve nonvolatile memory similar to that based on nano-floating-gate transistors. In addition, the density of the MPc core-specifically, charge-trapping sites, can be controlled by the length of the arm polymer, enabling control of the resultant memory device performance. ${ }^{34}$

As a continuation of our studies on the OFET memory devices, we herein attempt to develop a simple and easy method of preparing OFET memory devices using polystyrene-based nano-floating gates. We used our unique design of memory materialsstar-shaped polystyrene with specific charge-trapping sites-to prepare vertically phase-separated organic semiconductor/memory layers via a one-pot solution process. More specifically, the MPccored star-shaped polystyrene promotes the crystallization of small molecular semiconductors of TIPS-pentacene via polymer-matrixassisted phase-separation and simultaneously provides organic semiconductor/memory layers on the substrates. The morphology and structure of the blend films of the star polymer and TIPSpentacene prepared by a simple spin-coating process from various organic solvents were investigated using X-ray diffraction (XRD), atomic force microscopy (AFM), polarized optical microscopy, UVvis absorption spectroscopy, and X-ray photoelectron spectroscopy (XPS). We obtained highly crystalline and vertically phase-separated TIPS-pentacene on star polymer blend films from an orthodichlorobenzene (ODCB) solution, which is advantageous for OFET memory device applications. We finally demonstrated an OFET device with a bottom-gate top-contact configuration (Fig. 1a).
The resultant device showed a hole mobility of $0.1 \mathrm{~cm}^{2} \mathrm{~V}^{-1} \mathrm{~s}^{-1}$ and a high on/off current ratio greater than $10^{6}$. In addition, a memory window of $19.7 \mathrm{~V}$ was probed under an applied gate voltage in the range $\pm 50 \mathrm{~V}$ and the device demonstrated long chargeretention ability. Thus, the present one-pot solution process would be a useful approach for preparing non-volatile organic memories without cumbersome fabrication and costly vacuum-deposition processes of organic semiconductors.

\section{Experimental}

\section{Materials and methods}

Commercial chemicals, which were purchased from SigmaAldrich, TCI, Wako Chemicals, or Kanto Chemicals, were used without further purification unless noted otherwise. All reactions were conducted under Ar in oven-dried glassware.

\section{Measurements}

Electronic absorption spectra were recorded on a JASCO V-670 spectrophotometer. X-Ray diffraction (XRD) measurements were carried out using a Rigaku MiniFlex 600 (Ni-filtered $\mathrm{Cu} \mathrm{K}$ radiation, Rigaku, Tokyo, Japan). X-Ray photoelectron spectroscopy (XPS) was performed using a PHI Quantera SXM (ULVAC$\mathrm{PHI}$, Japan) with a monochromatic $\mathrm{Al} \mathrm{K} \alpha \mathrm{X}$-ray source. Atomic force microscopy (AFM) was performed under ambient conditions with a Bruker MultiMode 8 atomic force microscope. Imaging was conducted in the ScanAsyst mode using a silicon cantilever (NCHV-A) with a frequency of $270-400 \mathrm{kHz}$. The optical texture of the organic semiconductor crystals was analyzed by polarizing optical microscope (Olympus BX51N-31P-O3, Olympus, Tokyo, Japan), equipped with a DP22 digital camera.

\section{Device measurements}

The film thickness of each layer was measured using a microfigure measuring instrument (SURFCORDER ET200; Kosaka Laboratory, Tokyo). Devices were fabricated on highly doped $n$-type $\mathrm{Si}(100)$ wafers with a $300 \mathrm{~nm}$-thick $\mathrm{SiO}_{2}$ layer. In this a)
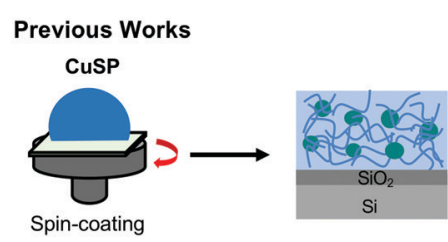

This Work

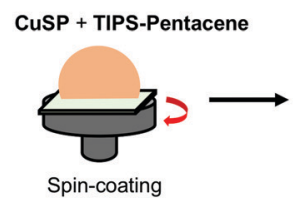

Spin-coating

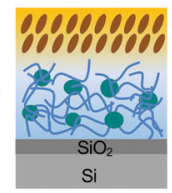

Vacuumdeposition of Pentacene$$
\text { . }
$$
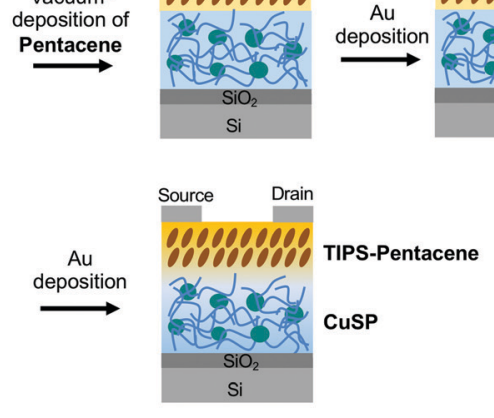

Fig. 1 (a) Schematic representation of the fabrication of OFET memory devices. (b) Chemical structures of TIPS-pentacene and polymer dielectrics of CuSP and $\mathrm{PS}_{4}$. 
study, all of the results for memory performance were averaged from at least ten devices from five batches. All measurements on the transistor memory devices were conducted with a Keithley 2636B source measurement unit under dark ambient conditions. Triaxial cables were connected to the probe station to minimize background noise. The effective charge carrier mobility $\left(\mu_{\text {eff }}\right)$ and threshold voltage $\left(V_{\text {th }}\right)$ values were estimated from the slope and intercept of the linear plot of the square root of the drain-to-source current $\left(I_{\mathrm{ds}}{ }^{1 / 2}\right)$ vs. the gate voltage $\left(V_{\mathrm{g}}\right)$ in the saturation regime using the following equation:

$$
I_{\mathrm{ds}}=W C_{\mathrm{tot}} \mu_{\mathrm{eff}}\left(V_{\mathrm{g}}-V_{\mathrm{t}}\right)^{2} /(2 L)
$$

where $C_{\text {tot }}$ is the capacitance per unit area of the total dielectric layer and $L$ and $W$ are the channel length and width, respectively.

The relations between the capacitance $\left(C_{\text {tot }}\right)$ of the device, $\mathrm{SiO}_{2}$ wafer $\left(C_{\mathrm{SiO} 2}\right)$, polymers $\left(C_{\text {poly }}\right)$, and polymer dielectric constant $(\varepsilon)$ are defined as

$$
\begin{gathered}
\frac{1}{C_{\mathrm{tot}}}=\frac{1}{C_{\mathrm{poly}}}+\frac{1}{C_{\mathrm{SiO}_{2}}} \\
C_{\text {poly }}=\frac{\varepsilon_{0} \varepsilon}{d}
\end{gathered}
$$

where $\varepsilon_{0}$ is the vacuum permittivity $\left(8.854 \times 10^{-12} \mathrm{~F} \mathrm{~m}^{-1}\right), d$ is the thickness of the dielectric, and $C_{\mathrm{SiO} 2}$ is $11.5 \mathrm{nF} \mathrm{cm}^{-2}$. The capacitances were calculated using eqn (2)-(3) in conjunction with the reported dielectric constants of 2.24 for CuSP and 2.48 for $\mathbf{P S}_{\mathbf{4}}$, respectively. ${ }^{33}$

\section{Results and discussion}

Structure of blend films of organic semiconductor and polymer dielectrics

The preparation of star-shaped polystyrene with a copper phthalocyanine core (CuSP) and a number-average molecular weight $\left(M_{n}\right)$ of $16.7 \mathrm{~kg} \mathrm{~mol}{ }^{-1}$ has been previously reported. ${ }^{33}$ Four-armed polystyrene without the CuPc core $\left(\mathbf{P S}_{\mathbf{4}}\right)\left(M_{n}=\right.$ $20.5 \mathrm{~kg} \mathrm{~mol}^{-1}$ ) was also prepared in the present work as a reference compound. TIPS-pentacene, a derivative of pentacene, was selected as a soluble organic semiconductor, and the device performance was compared with that reported in previous studies in which vacuum-deposited pentacene was used as an organic semiconductor. The chemical structures of all the compounds studied in the present work are shown in Fig. 1b. First, CuSP and TIPS-pentacene were dissolved in organic solvents at a concentration of $10 \mathrm{mg} \mathrm{mL}$; each solution was then mixed in a 1:1 volume ratio and stirred for $2 \mathrm{~h}$ under ambient conditions. The mixture was spin-coated at $1000 \mathrm{rpm}$ onto a Si wafer substrate; the resultant films were vacuum dried to remove any remaining solvent. The film morphology was strongly influenced by the choice of solvent. Therefore, to obtain an ideal film morphology for OFET memory device applications, we tested three different solvents-chloroform $\left(\mathrm{CHCl}_{3}\right)$, toluene, and ODCB-for preparing the films. Blend films were also prepared from a mixture of $\mathbf{P S}_{\mathbf{4}}$ and TIPS-pentacene for
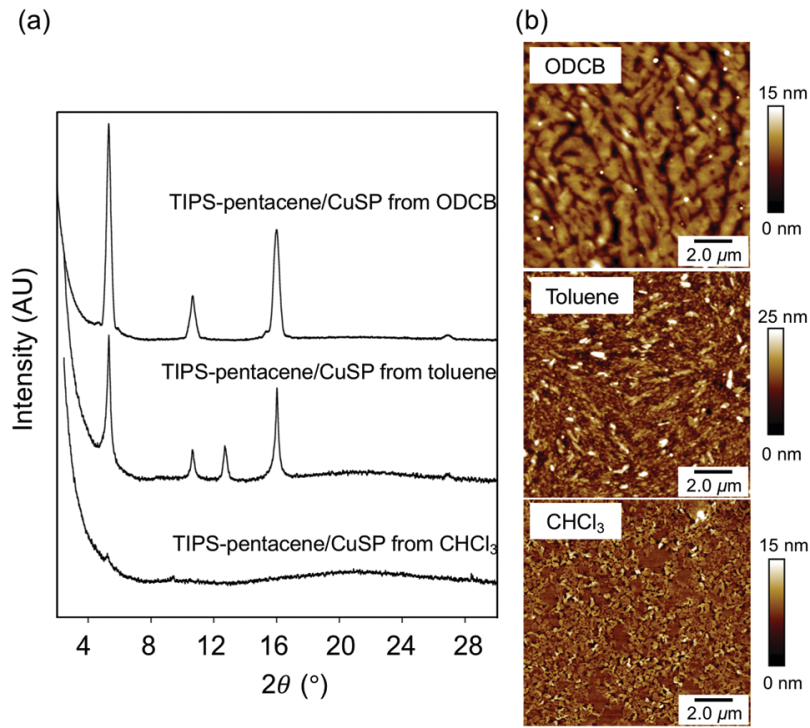

Fig. 2 (a) XRD profiles of TIPS-pentacene/CuSP blend films spin-coated from various solvents. (b) AFM height images of the TIPS-pentacene/CuSP blend films.

comparison. The thicknesses of the TIPS-pentacene/polymer films spin-coated from $\mathrm{CHCl}_{3}$, toluene, and ODCB were found to be approximately 100,60 , and $50 \mathrm{~nm}$, respectively.

The spin-coated blend films were analyzed by X-ray diffraction (XRD) to characterize the structure of the TIPS-pentacene in the blend film. As shown in Fig. 2a, the out-of-plane XRD patterns of the mixed film obtained from ODCB exhibited strong and sharp diffraction peaks corresponding to the crystal lattice of the (001) planes of TIPS-pentacene, indicating a crystalline structure parallel to the substrate, with an interplanar $d$-spacing of $16.3 \AA$ estimated from the peak at $2 \theta=5.36^{\circ}$. This result indicates that the triisopropylsilylethynyl groups of the TIPS-pentacene molecules align in the edge-on orientation with respect to the substrates, consistent with a single crystal of TIPS-pentacene with a domain spacing of $16.8 \AA^{35,36}$ The XRD pattern of the blend film spin-coated from toluene solution showed an additional peak at a $d$-spacing of $6.92 \AA$, indicating that the crystals oriented in directions other than (001) were included in the mixture. The XRD pattern of the film prepared from $\mathrm{CHCl}_{3}$ did not show clear peaks; instead, the amorphous halo originating from the polymer chains was observed at $2 \theta \approx$ $20^{\circ}$. The surface morphology analyzed using atomic force microscopy (AFM) supported these results (Fig. 2b). The large continuous crystal grains of TIPS-pentacene were observed in the blend films spin-coated from ODCB, whereas small needle-like crystals with a size of $\sim 0.5 \mu \mathrm{m}$ and a random orientation were observed in the blend film spin-coated from toluene. The film from $\mathrm{CHCl}_{3}$ exhibited a relatively smooth surface without large crystal domains. These results reveal that the crystallization of the TIPS-pentacene in the star-polymer matrix was highly dependent on the solvent, mainly because of the difference in the solvents' evaporation rate. Slow evaporation of an ODCB solution induced phase-separation of the TIPS-pentacene and star-shaped polymer, promoting crystallization of the TIPS- 
pentacene, whereas fast evaporation inhibited the formation of crystals in the case of the film spin-coated from $\mathrm{CHCl}_{3}$ solution. Approximately the same XRD patterns and AFM images were obtained for the blend films prepared using $\mathbf{P S}_{\mathbf{4}}$ as the polymer matrix, implying that the CuPc core in CuSP did not disturb the crystallization of the TIPS-pentacene (Fig. S1 and S2, ESI $\dagger$ ).

The blend films on quartz plates were also analyzed by polarized optical microscopy (POM). As shown in Fig. 3a, large spherulite textures were observed for the blend films spincoated from ODCB. The blend film spin-coated from a toluene solution showed smaller spherulite textures; the film spincoated from $\mathrm{CHCl}_{3}$ did not show large crystals. The different morphologies of the TIPS-pentacene in the blend films spincoated from the various solvents were also examined by UV-vis absorption spectroscopy (Fig. 3b). The absorption spectrum of TIPS-pentacene in $\mathrm{C} \mathrm{CHCl}_{3}$ solution showed three characteristic absorption bands in the 500-700 $\mathrm{nm}$ region; these bands originated from the first excited singlet state. A similar spectrum was observed for a film spin-coated from a $\mathrm{CHCl}_{3}$ solution of TIPS-pentacene/CuSP, indicating that, like the TIPSpentacene in the solution state, the most of the TIPS-pentacene molecules in the polymer film existed in monomeric form. The fast evaporation of $\mathrm{CHCl}_{3}$ solution during the spin-coating process might disperse the TIPS-pentacene molecules in the polymer matrix before crystallization proceeded. An additional shoulder band at $\sim 700 \mathrm{~nm}$ was observed in the spectrum of the film obtained from toluene solution, which originated from the aggregated structure of TIPS-pentacene. The spectrum of the blend film spin-coated from ODCB solution showed broader absorption bands at approximately 600, 650, and $700 \mathrm{~nm}$. These results reveal that most of the TIPS-pentacene molecules assembled to form crystals even in the presence of the polymer matrix. Notably, the absorbance from the CuPc core at $\sim 630 \mathrm{~nm}$ in the spectra of the blend films was (a)

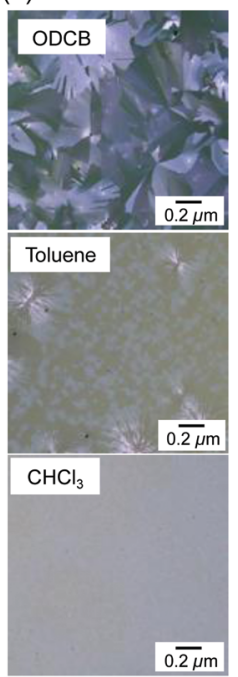

(b)

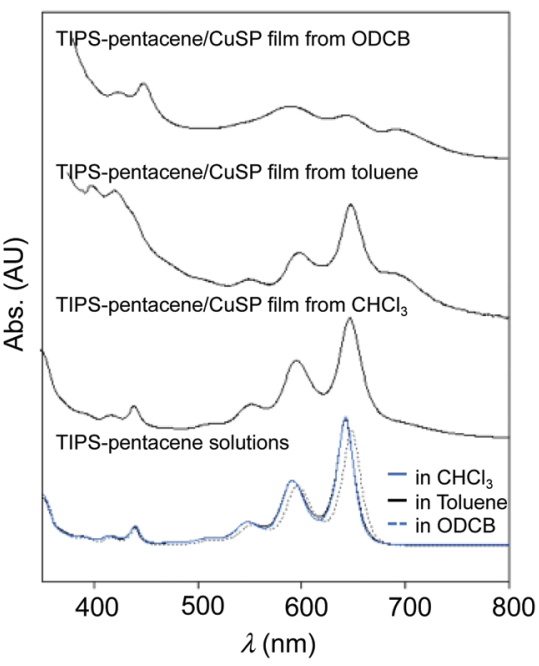

Fig. 3 (a) POM images of the TIPS-pentacene/CuSP blend films. (b) Absorption spectra of TIPS-pentacene/CUSP blend films and TIPSpentacene solutions. (a)

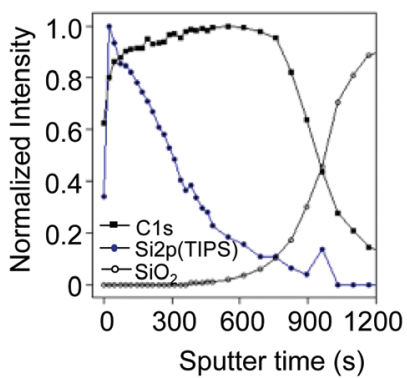

(b)

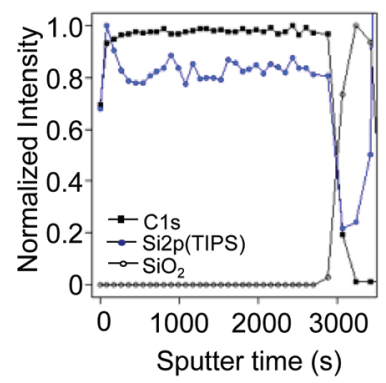

Fig. 4 XPS depth profiles of a TIPS-P/CuSP blend films coated from (a) $\mathrm{ODCB}$ and (b) $\mathrm{CHCl}_{3}$ solutions on a Si substrate.

overlapped by the strong absorption of the TIPS-pentacene because of the small amount of CuPc unit in the polymer matrix.

We used XPS to investigate the detailed structure in the blend films of TIPS-pentacene and CuSP spin-coated from ODCB and $\mathrm{CHCl}_{3}$ on the Si wafer substrates. The depth profiles of the film were collected by $\mathrm{Ar}^{+}$-ion sputtering with an etching rate of $2.7 \mathrm{~nm} \mathrm{~min}{ }^{-1}$ for the film from ODCB and $3.2 \mathrm{~nm} \mathrm{~min} \mathrm{~m}^{-1}$ for the film from a $\mathrm{CHCl}_{3}$ solution. All XPS peaks were referenced to the neutral C 1s peak at a binding energy of $284.8 \mathrm{eV}$. The peak intensities at binding energies corresponding to $\mathrm{C} 1 \mathrm{~s}$ and Si 2p were plotted against the sputter time (Fig. 4). Notably, the Si $2 p$ binding energies in the spectra of the TIPS-pentacene and $\mathrm{SiO}_{2}$ on the Si-wafer substrates were sufficiently different for each component to be identified (Fig. S3, ESI $\dagger$ ). In addition, the peaks of $\mathrm{N} 1 \mathrm{~s}$ or $\mathrm{Cu} 2 \mathrm{p}$ in the spectra of CuSP were difficult to observe because of the small atom\%. The depth profiles of the blend film spin-coated from ODCB indicate that the TIPSpentacene phase was located at the upper interface and mainly existed to a depth of $\sim 20 \mathrm{~nm}$ from the top interface in a blend film with a total thickness of $\sim 50 \mathrm{~nm}$. This result is consistent with the aforementioned AFM analyses. On the other hand, the peak intensities of Si $2 \mathrm{p}$ corresponding to TIPS-pentacene in the $\mathrm{CHCl}_{3}$-processed blend film were relatively constant throughout the film thickness. This result indicated that the TIPS-pentacene molecules were distributed in the polymer film without clear phase segregation, which also agreed with the above results. We concluded that the TIPS-pentacene forms large crystals on the CuSP polymer layer via vertical phase segregation of the blend film deposited by a simple one-pot spin-coating process from ODCB solution.

\section{OFET characteristics}

Having successfully prepared vertically segregated organic semiconductor and polymer layers on the substrates via a one-pot solution process, we further fabricated OFET devices with a bottom-gate/top-contact configuration (Fig. 1a). Topcontact gold electrodes with a channel length $(L)$ of $50 \mu \mathrm{m}$ and a width $(W)$ of $1000 \mu \mathrm{m}$ were deposited onto blend films of TIPS-pentacene and star polymers of CuSP or $\mathbf{P S}_{\mathbf{4}}$ spin-coated onto highly doped n-type $\mathrm{Si}(100)$ wafers with a $300 \mathrm{~nm}$-thick surface layer of $\mathrm{SiO}_{2}$. The capacitance of the gate dielectric $\left(C_{\text {tot }}\right)$ was estimated on the basis of the reported dielectric constants 
of $\mathbf{C u S P}$ or $\mathbf{P S}_{\mathbf{4}}$. From the aforementioned results, the thickness of the polymer dielectrics was assumed to be $30 \mathrm{~nm}$ given that the total thickness of the blend film was $50 \mathrm{~nm}$ and the TIPSpentacene crystals were $20 \mathrm{~nm}$ thick.

The electrical transfer and output characteristics of the fabricated OFET devices are shown in Fig. S4 (ESI $\dagger$ ). The reliability factors (r) for $\mu_{\text {eff }}$ of the OFET devices with CuSP and $\mathbf{P S}_{\mathbf{4}}$ were also estimated (Fig. S4, ESI $\dagger$, inset). ${ }^{37}$ When the gate bias was scanned between +10 and $-50 \mathrm{~V}$, the typical p-type accumulation mode was observed for the blend films spin-coated from ODCB. The devices with the blend films of TIPS-pentacene and CuSP or $\mathbf{P S}_{\mathbf{4}}$ spin-coated from ODCB showed average $\mu_{\text {eff }}$ values of $0.10 \pm 0.05$ and $0.11 \pm 0.09 \mathrm{~cm}^{2} \mathrm{~V}^{-1} \mathrm{~s}^{-1}$, respectively. Compared to the reported $\mu_{\text {eff }}$ values of $0.21 \mathrm{~cm}^{2} \mathrm{~V}^{-1} \mathrm{~s}^{-1}$ for vacuum-deposited pentacene-embedded OFET, ${ }^{34}$ these values are sufficiently high for solution-processed OFET devices. The on/off ratios of the drain current $\left(I_{\text {on }} / I_{\text {off }}\right)$ were greater than $10^{6}$, which is beneficial for the memory device to clearly distinguish between the " 0 ", and " 1 " digital states. As previously discussed, large crystal grains of TIPS-pentacene on the polymer dielectrics provided efficient charge transport pathways in the OFET devices. By contrast, the OFET device with the blend film spin-coated from toluene showed a low hole mobility of $1.0 \times 10^{-3} \mathrm{~cm}^{2} \mathrm{~V}^{-1} \mathrm{~s}^{-1}$ at the maximum (Fig. S5a and b, ESI $\dagger$ ). In addition, the OFET devices with embedded blend films coated from $\mathrm{CHCl}_{3}$ did not demonstrate transistor characteristics (Fig. S5c and d, ESI $\dagger$ ). The lack of large TIPS-pentacene crystals or the polymer insulating layer on the surface disturbed the efficient charge transport in the OFET devices.

\section{Memory characteristics}

We confirmed that the blend film of TIPS-pentacene and CuSP prepared via a one-pot solution process showed vertical phase separation between the organic semiconductor and the polymer dielectrics and that this phase separation led to facile crystallization of the organic semiconductor, which is essential for high-performance OFET devices. The polymer dielectrics of CuSP function not only as a matrix but also as a memory material because the polymer has distinct charge-trapping sites, i.e., CuPc cores, that can trap hole charges upon application of a negative gate voltage. To investigate the memory properties of the CuSP-embedded OFETs, we operated the devices by alternately switching the gate bias $\left(V_{\mathrm{g}}\right)$ between -50 and $+50 \mathrm{~V}$ as a pulse stimulus with a fixed drain voltage $\left(V_{\mathrm{d}}\right)$ of $-50 \mathrm{~V}$. As shown in Fig. 5a and Fig. S6 (ESI $\dagger$ ), the transfer curve exhibited a negative shift after a negative gate voltage of $V_{\mathrm{g}}=-50 \mathrm{~V}$ was applied for $1 \mathrm{~s}$ (writing process). This result indicates that the hole charges accumulated in the TIPS-pentacene layer were injected and stored in the underlying CuSP layer, resulting in a built-in electric field opposite the applied $V_{\mathrm{g}}$. The efficient injection of charge into the CuPc core in the polymer layer was achieved via the appropriate highest occupied molecular orbital (HOMO) energy levels of the CuSP and TIPS-pentacene of -5.2 and $-5.1 \mathrm{eV}$, respectively (Fig. S7, ESI $\dagger$ ). Conversely, upon application of a positive gate bias of $+50 \mathrm{~V}$ to the OFET device, the transfer curve shifted in the positive direction, returning to

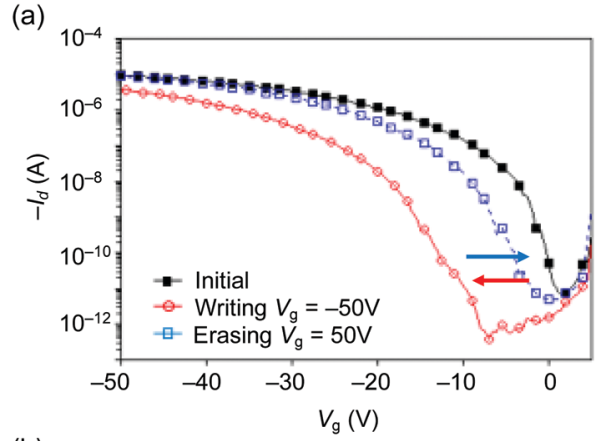

(b)

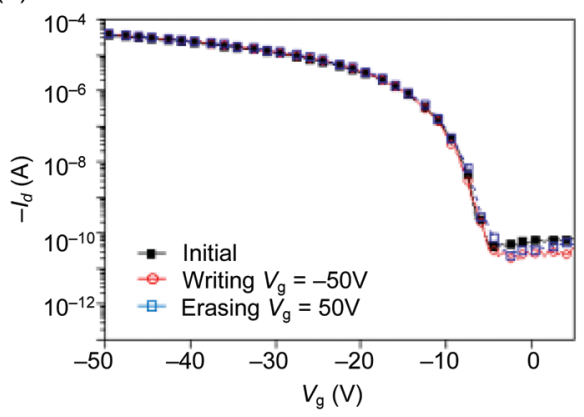

Fig. 5 Transfer characteristics of OFET memory devices fabricated using (a) TIPS-P/CuSP blend films and (b) TIPS-P/PS 4 blend films monitored at $V_{d}=-50 \mathrm{~V}$.

its initial position. This operation is referred to as the erasing process, where the stored hole charges in CuSP are transferred back to the channel, implying that the device is categorized as a flash-type memory device. One may concern here the possibility of charge trapping in TIPS-pentacene molecules buried in the polymer layer. However, the OFET embedding a blend film of TIPS-pentacene and $\mathbf{P S}_{\mathbf{4}}$ without CuPc core did not show any shift in the threshold voltage upon writing and erasing processes (Fig. 5b). This result confirms that the memory characteristics were derived from the CuPc core in the star polymer layer. The shift of $V_{\mathrm{th}}\left(\Delta V_{\mathrm{th}}\right)$, which is referred to as the memory window, was evaluated to be $19.7 \pm 5.5 \mathrm{~V}$, which is similar to the reported memory window of $\Delta V_{\mathrm{th}}=21 \pm 1.03 \mathrm{~V}$ for a vacuum-deposited pentacene-based OFET memory device with embedded CuSP. ${ }^{33}$ The maximum memory ratio (i.e., the $I_{\mathrm{on}} / I_{\text {off }}$ ratio at the reading gate voltage after the writing/erasing process) was $\sim 10^{3}$ because of the intrinsic large $I_{\text {on }} / I_{\text {off }}$ value of the device (i.e., $10^{6}$ ). The (a)

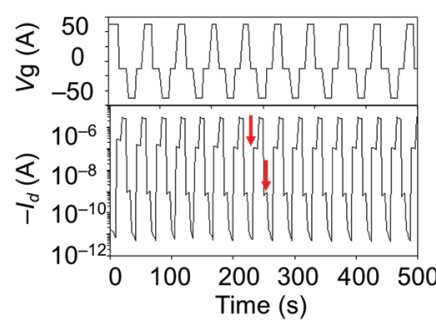

(b)

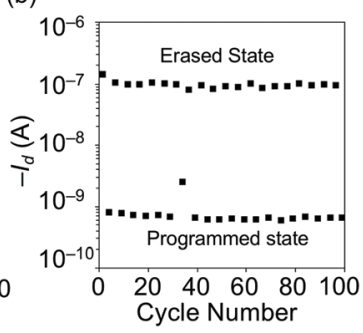

Fig. 6 WRER test (a) and endurance characteristics (b) of the OFET memory device with TIPS-pentacene/CuSP blend films. 


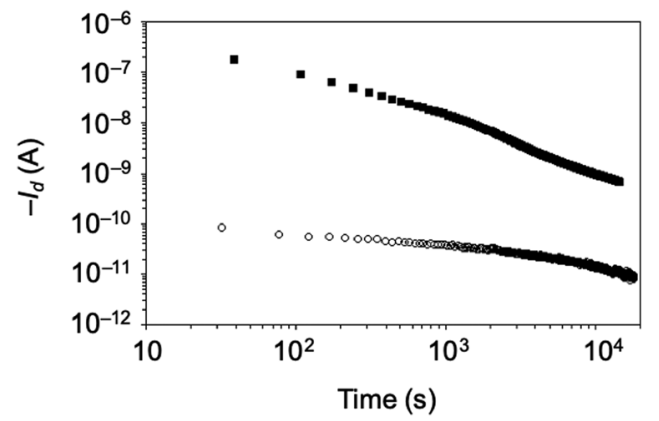

Fig. 7 Retention time of the $I_{d}$ of the OFET memory device with TIPSpentacene/CuSP blend films, as monitored at $V_{\mathrm{g}}=-5 \mathrm{~V}$ after writing (black squares) and erasing (white circles) operations.

multiple switching durability of the OFET with CuSP was further evaluated through write-read-erase-read (WRER) cycle test. The drain current was read at $V_{\mathrm{g}}=-10 \mathrm{~V}$ with the fixed $V_{\mathrm{d}}=-50 \mathrm{~V}$ after repeating the writing and erasing operations with the continuous bias pluses of $-50 \mathrm{~V} / 50 \mathrm{~V}$ (Fig. 6a). After several cycles, the $I_{\mathrm{ON}} / I_{\mathrm{OFF}}$ ratio was maintained above $10^{2}$ over 100 cycles (Fig. 6b).

Charge retention is also important when evaluating the nonvolatility of a memory device. To evaluate the memory retention characteristics, we monitored the drain current at $V_{\mathrm{g}}=-5 \mathrm{~V}$ after a writing operation at $V_{\mathrm{g}}=-50 \mathrm{~V}$ for $1 \mathrm{~s}$ and an erasing operation at $V_{\mathrm{g}}=+50 \mathrm{~V}$ for $1 \mathrm{~s}$ (Fig. 7). The difference in the drain on/off current was maintained at $10^{2}$ for $12000 \mathrm{~s}$. High stability of the stored charge was achieved because of the unique architecture of the star-shaped polymer, where the arm polymer chains surrounding the CuPc core assemblies restrain the leakage of the trapped charges.

\section{Conclusions}

We have successfully developed high-performance solutionprocessed OFET memory devices using TIPS-pentacene and copper phthalocyanine-cored star-shaped polystyrene. The morphology and crystal structure of the TIPS-pentacene in the polymer blend film was found to be dependent on the solvent. We also found that the TIPS-pentacene/polymer film spincoated from ODCB solution showed vertical phase separation, with large crystal grains of the organic semiconductor at the top surface, which is advantageous for OFET device applications. The OFET devices fabricated by the solution process using the blend films of TIPS-pentacene and CuSP exhibited a high charge carrier mobility with substantial memory characteristics. The novel methodology of using a polymer nano-floating gate to construct OFET memory in a one-pot solution process is a promising technique to prepare high-performance OFET memory devices and would be useful for fabricating flexible and large-area memory devices.

\section{Conflicts of interest}

There are no conflicts to declare.

\section{Acknowledgements}

We are grateful to Dr Hideyuki Yasufuku in Research Network and Facility Services Division (RNFS), NIMS for the XPS measurements and analyses. This work was partially supported by JSPS KAKENHI Grant (JP 21K05220, 18K14300), The Murata Science Foundation, and the Iketani Science and Technology Foundation. C.-F.H. acknowledges the support from the Ministry of Science and Technology, Taiwan (MOST108-2923E-005-001-MY2).

\section{References}

1 T. Sekitani, T. Yokota, U. Zschieschang, H. Klauk, S. Bauer, K. Takeuchi, M. Takamiya, T. Sakurai and T. Someya, Science, 2009, 326, 1516-1519.

2 K. J. Baeg, D. Khim, J. Kim, B. D. Yang, M. Kang, S. W. Jung, I. K. You, D. Y. Kim and Y. Y. Noh, Adv. Funct. Mater., 2012, 22, 2915-2926.

3 S. J. Kim and J. S. Lee, Nano Lett., 2010, 10, 2884-2890.

4 W. L. Leong, N. Mathews, B. Tan, S. Vaidyanathan, F. Dotz and S. Mhaisalkar, J. Mater. Chem., 2011, 21, 5203-5214.

5 M. Kang, K. J. Baeg, D. Khim, Y. Y. Noh and D. Y. Kim, Adv. Funct. Mater., 2013, 23, 3503-3512.

6 C. C. Shih, W. Y. Lee and W. C. Chen, Mater. Horiz., 2016, 3, 294-308.

7 L. V. Tho, K. J. Baeg and Y. Y. Noh, Nano Convergence, 2016, 3, 10.

8 J. S. Lee, Electron. Mater. Lett., 2011, 7, 175-183.

9 W. L. Leong, N. Mathews, B. Tan, S. Vaidyanathan, F. Dotz and S. Mhaisalkar, J. Mater. Chem., 2011, 21, 8971-8974.

10 Y. H. Chou, Y. C. Chiu and W. C. Chen, Chem. Commun., 2014, 50, 3217-3219.

11 C. Wu, W. Wang and J. F. Song, Appl. Phys. Lett., 2016, 109.

12 W. Li, F. N. Guo, H. F. Ling, H. Liu, M. D. Yi, P. Zhang, W. J. Wang, L. H. Xie and W. Huang, Small, 2018, 14.

13 Y. Park, K. J. Baeg and C. Kim, ACS Appl. Mater. Interfaces, 2019, 11, 8327-8336.

14 F. Shiono, H. Abe, T. Nagase, T. Kobayashi and H. Naito, Org. Electron., 2019, 67, 109-115.

15 V. Coropceanu, J. Cornil, D. A. da Silva, Y. Olivier, R. Silbey and J. L. Bredas, Chem. Rev., 2007, 107, 926-952.

16 T. Ohe, M. Kuribayashi, R. Yasuda, A. Tsuboi, K. Nomoto, K. Satori, M. Itabashi and J. Kasahara, Appl. Phys. Lett., 2008, 93, 053303.

17 J. Kang, N. Shin, D. Y. Jang, V. M. Prabhu and D. Y. Yoon, J. Am. Chem. Soc., 2008, 130, 12273.

18 R. Hamilton, J. Smith, S. Ogier, M. Heeney, J. E. Anthony, I. McCulloch, J. Veres, D. D. C. Bradley and T. D. Anthopoulos, Adv. Mater., 2009, 21, 1166-1171.

19 J. Smith, R. Hamilton, I. McCulloch, N. StingelinStutzmann, M. Heeney, D. D. C. Bradley and T. D. Anthopoulos, J. Mater. Chem., 2010, 20, 2562-2574.

20 L. H. Chou, Y. Na, C. H. Park, M. S. Park, I. Osaka, F. S. Kim and C. L. Liu, Polymer, 2020, 191.

21 L. Janasz, M. Borkowski, P. W. M. Bolm, T. Marszalek and W. Pisula, Adv. Funct. Mater., 2021, 2105456. 
22 M. B. Madec, D. Crouch, G. R. Llorente, T. J. Whittle, M. Geoghegan and S. G. Yeates, J. Mater. Chem., 2008, 18, 3230-3236.

23 D. K. Hwang, C. Fuentes-Hernandez, J. D. Berrigan, Y. N. Fang, J. Kim, W. J. Potscavage, H. Cheun, K. H. Sandhage and B. Kippelen, J. Mater. Chem., 2012, 22, 5531-5537.

24 N. Shin, J. Kang, L. J. Richter, V. M. Prabhu, R. J. Kline, D. A. Fischer, D. M. DeLongchamp, M. F. Toney, S. K. Satija, D. J. Gundlach, B. Purushothaman, J. E. Anthony and D. Y. Yoon, Adv. Funct. Mater., 2013, 23, 366-376.

25 M. R. Niazi, R. P. Li, E. Q. Li, A. R. Kirmani, M. Abdelsamie, Q. X. Wang, W. Y. Pan, M. M. Payne, J. E. Anthony, D. M. Smilgies, S. T. Thoroddsen, E. P. Giannelis and A. Amassian, Nat. Commun., 2015, 6, 8598.

26 T. Makita, M. Sasaki, T. Annaka, M. Sasaki, H. Matsui, C. Mitsui, S. Kumagai, S. Watanabe, T. Hayakawa, T. Okamoto and J. Takeya, Appl. Phys. Lett., 2017, 110.

27 M. Lada, M. J. Starink, M. Carrasco, L. C. Chen, P. Miskiewicz, P. Brookes, M. Obarowska and D. C. Smith, J. Mater. Chem., 2011, 21, 11232-11238.

28 W. H. Lee, D. Kwak, J. E. Anthony, H. S. Lee, H. H. Choi, D. H. Kim, S. G. Lee and K. Cho, Adv. Funct. Mater., 2012, 22, 267-281.
29 A. M. Gaikwad, Y. Khan, A. E. Ostfeld, S. Pandya, S. Abraham and A. C. Arias, Org. Electron., 2016, 30, 18-29. 30 J. H. Lee, Y. Seo, Y. D. Park, J. E. Anthony, D. H. Kwak, J. A. Lim, S. Ko, H. W. Jang, K. Cho and W. H. Lee, Sci. Rep., 2019, 9, 21.

31 K. Kim, J. Hong, S. G. Hahm, Y. Rho, T. K. An, S. H. Kim and C. E. Park, ACS Appl. Mater. Interfaces, 2019, 11, 13481-13490.

32 Y. Diao, B. C. K. Tee, G. Giri, J. Xu, D. H. Kim, H. A. Becerril, R. M. Stoltenberg, T. H. Lee, G. Xue, S. C. B. Mannsfeld and Z. N. Bao, Nat. Mater., 2013, 12, 665-671.

33 J. Aimi, C. T. Lo, H. C. Wu, C. F. Huang, T. Nakanishi, M. Takeuchi and W. C. Chen, Adv. Electron. Mater., 2016, 2, 1500300.

34 J. Aimi, P. H. Wang, C. C. Shih, C. F. Huang, T. Nakanishi, M. Takeuchi, H. Y. Hsueh and W. C. Chen, J. Mater. Chem. C, 2018, 6, 2724-2732.

35 J. H. Chen, J. Anthony and D. C. Martin, J. Phys. Chem. B, 2006, 110, 16397-16403.

36 G. Giri, E. Verploegen, S. C. B. Mannsfeld, S. Atahan-Evrenk, D. H. Kim, S. Y. Lee, H. A. Becerril, A. Aspuru-Guzik, M. F. Toney and Z. A. Bao, Nature, 2011, 480, 504-508.

37 M. Sugiyama, S. Jancke, T. Uemura, M. Kondo, Y. Inoue, N. Namba, T. Araki, T. Fukushima and T. Sekitani, Org. Electron., 2021, 96, 106219. 